\title{
A QUANTITATIVE ANALYSIS OF THE KINETICS OF CHOLINESTERASE INHIBITION IN TISSUE HOMOGENATES OF MICE AND HOUSEFLIES
}

\author{
K. VAN ASPEREN AND H. M. DEKHUIJZEN \\ Laboratory for Research on Insecticides, Utrecht* (The Netherlands)
}

In a previous paper ${ }^{1}$ we reported on the remarkable difference between the cholinesterases $(\mathrm{ChE})$ in homogenates of mouse brain and housefly heads with respect to inhibition by the organophosphorus insecticide DDVP (O,O-Dimethyl-O-2,2-dichlorovinyl phosphate). It was found that the inhibitor concentration giving $50 \%$ inhibition of ChE-activity is about $\mathrm{ro}^{-7} M$ in the case of mouse-brain suspensions and approximately $10^{-9} M$ in the case of fly-head homogenates. It was suggested that difference in susceptibility to DDVP-inhibition need not necessarily mean that the enzymes themselves are chemically different, since such a difference could just as well be caused by other factors, e.g. binding of the inhibitor to proteins. It is the purpose of the present paper to give a further quantitative analysis of the kinetics of the inhibitory reaction.

According to ALDRIDGE ${ }^{2}$ (see also CASIDA $^{3}$ ) the first reaction to occur between organophosphates and $\mathrm{ChE}$ is the formation of an unstable complex, which then dissociates (reaction (2)) to yield an alcoholic, phenolic, or acidic compound and the phosphorylated enzyme. The phosphorylation blocks the active (esteratic) site of the enzyme and hydrolysis of the phosphorylated enzyme (reaction (3)) is a very slow process. In general, therefore, the inhibition by organophosphorus compounds is practically irreversible or only slowly reversible, the reversibility depending on the resistance of the phosphorylated enzyme to hydrolysis. In the case of DDVP, the ultimate fate of the DDVP-molecule will be its hydrolysis to dichlorovinyl alcohol (V) and dimethyl phosphoric acid (P). This latter product, however, remains combined with the enzyme and thus inactivates it ( $\mathrm{EP}=$ the phosphorylated enzyme). It will be released from the enzyme only very slowly by hydrolysis (reaction (3)). The first reaction is reversible; reactions (2) and (3) are irreversible.

The simplest formulation of the reaction sequence with the corresponding equations for equilibrium constant $K_{i}$ and reaction velocities $v_{1}$ and $v_{2}$ seems to be as follows:

$$
\begin{array}{ll}
\mathrm{E}+\mathrm{I} \leftrightarrows \mathrm{EI} & K_{i}=\frac{[\mathrm{E}] \cdot[\mathrm{I}]}{[\mathrm{EI}]} \\
\mathrm{EI} \rightarrow \mathrm{EP}+\mathrm{V} & v_{1}=k_{1} \cdot[\mathrm{EI}] \\
\mathrm{EP} \rightarrow \mathrm{E}+\mathrm{P} & v_{\mathrm{g}}=k_{2} \cdot[\mathrm{EP}]
\end{array}
$$

* Postal address: Vondellaan 6, Utrecht (The Netherlands). References p. $6 \times 3$. 
After addition of substrate the phosphorylated enzyme (EP) is inactive; the enzymeinhibitor complex EI, however, dissociates and the enzyme is available for combination with and hydrolysis of the substrate:

$$
\mathrm{EI}+\mathrm{S} \rightarrow \mathrm{ES}+\mathrm{I}
$$

Thus, the amount of potentially active enzyme $\left(E_{a c t}\right)$ is given by:

$$
\left[\mathrm{E}_{\mathrm{act}}\right]=[\mathrm{E}]+[\mathrm{EI}]
$$

When EP is stable $\left(k_{2}=0\right)$ the ultimate inhibition value will depend only on the ratio of the enzyme and inhibitor concentrations originally present in the reaction mixture. The $I_{50}$ value, i.e. the inhibitor concentration causing $50 \%$ inhibition of enzyme activity, will theoretically be half the enzyme concentration, and inhibition values are directly proportional to inhibitor concentrations, assuming that the incubation time, i.e. the time available for the reaction between enzyme and inhibitor before addition of substrate, is sufficiently long. The inhibition will proceed with second-order reaction kinetics.

When, however, the phosphorylated enzyme EP is hydrolysed with a reasonable velocity, the inhibition will reach a constant level as soon as reaction (2) and reaction (3), proceed with equal velocity, assuming $[\mathrm{I}] \gg[\mathrm{E}]$. When $[\mathrm{I}] \gg[\mathrm{E}]$, then $[\mathrm{I}]$ represents the total inhibitor concentration used, since only a negligible quantity of the inhibitor will be bound to the enzyme. It is then possible to derive the relation between inhibitor concentration and inhibition value, starting from the equations (I), (2), (3) and (4) given above.

[EI] may be calculated from equations (I) and (4):

$$
[\mathrm{EI}]=\frac{\left[\mathrm{E}_{\mathrm{act}}\right]}{\mathrm{I}+\frac{\kappa_{i}}{[\mathrm{I}]}}
$$

Constant inhibition is reached when $v_{1}=v_{2}$.

Then

$$
k_{1} \times \frac{\left[\mathrm{E}_{\mathrm{aet}}\right]}{1+\frac{K_{i}}{[\mathrm{I}]}}=k_{2} \times[\mathrm{EP}]
$$

Substituting $k_{3}$ for $k_{1} k_{2}$ we get:

$$
\frac{k_{3} \times[\mathrm{I}]}{[\mathrm{I}]+K_{i}}=\frac{\left[\mathrm{E} \mathrm{P}^{\mathrm{s}}\right]}{\left[\mathrm{E}_{\mathrm{act}}\right]}
$$

When the fractional activity (the fraction of the enzymic activity, which is not inhibited) is called $\alpha$, then

$$
\frac{[\mathrm{EP}]}{\left[\mathbf{E}_{\mathrm{act}}\right]}=\frac{\mathrm{I}-\boldsymbol{a}}{\alpha}
$$

and

$$
[\mathrm{I}] \times \frac{\alpha}{\mathrm{I}-\alpha}=\frac{[\mathrm{I}]+K_{i}}{h_{3}}
$$

$[I] \times \alpha /(\mathbf{I}-\alpha)$ will be approximately constant, when $[\mathrm{I}] \ll K_{i}$, which implies that $k_{3}$ will be large. This constancy of $[\mathrm{I}] \times \alpha /(\mathrm{I}-\alpha)$ holds also for a simple reversible inhibition process (see MiSSART ${ }^{4}$ ).

Rejerences p. 613 . 
Fig. I gives a schematic picture of the course of the reaction in the case of a slowly reversible inhibition as described above. Curve 2 represents the amount of enzyme, that has been phosphorylated

$$
\mathrm{E}+\mathrm{I} \leftrightarrows \mathrm{EI} \rightarrow \mathrm{EP}+\mathrm{V} .
$$

Curve 3 represents the amount of enzyme, that has been dephosphorylated

$$
\mathrm{EP} \rightarrow \mathrm{E}+\mathrm{P} .
$$

Fig. I. Schematic picture of a slowly reversible inhibition-reaction.

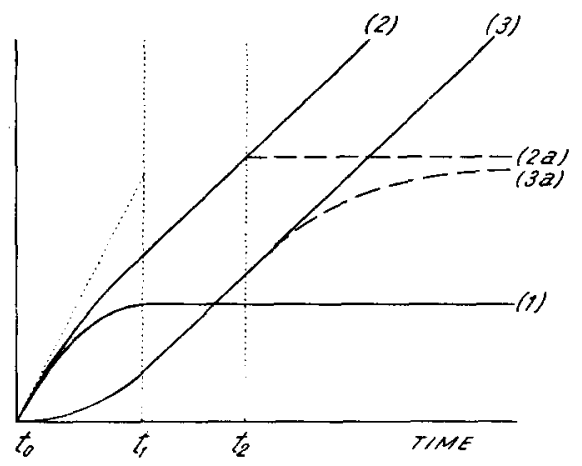

The vertical distance between the two curves represents the amount of phosphorylated enzyme EP present at any time. The course of the inhibition process, which can be experimentally tested, is represented by curve I. This curve plots the amounts of phosphorylated enzyme (vertical distance between curves 2 and 3 ) against time. The slope of curve 2 is proportional to the amount of nonphosphorylated enzyme and clecreases with time. The slope of curve 3 is proportional to the amount of phosphorylated enzymes and increases with time. At time $t_{1}$, equilibrium is reached and the slopes are equal. When equilibrium has been reached and the substrate (acetylcholine) then added (at time $t_{2}$ in the figure), reaction (2) is stopped and curve 2 will proceed horizontally (2a). Reaction (3), represented by curve 3 , will then proceed at a velocity that will decrease exponentially (3a).

When, at time $t_{1}$, the fractional activity is $a$, then the slope $\left(S_{0}\right)$ of curve 2 at time $t_{0}$ and the slopes $\left(S_{1}\right)$ of curves 2 and 3 at time $t_{1}$, will be interrelated by the following equation:

$$
S_{1}=a \times S_{0}
$$

(in the figure $\alpha$ has been taken as $\mathbf{I} / 2$ ).

Some elements and assumptions inserted in this picture will be dealt with later.

We shall now examine how the results of our investigations with homogenates of mouse brain and fly head fit into the more or less theoretical picture given above.

\section{METHOD}

The (hE-activity was measured manometrically with the Warburg-apparatus at $\mathrm{pH} 7.4(5 \%$ $\mathrm{CO}_{2}+95 \% \mathrm{~N}_{2}$ gas mixture $0 \mathrm{cr}$ saline containing $0.025 M \mathrm{NaHCO}_{3}$ and $\left.0.5 . M \mathrm{NaCl}\right)$ and $37^{\circ}$. Enzyme preparations consisted of homogenates prepared from freshly excised mouse-brain tissue (approx. $15 \mathrm{mg}$ of tissue $/ 2 \mathrm{ml}$ of saline in a Varburg-vessel) or from freshly obtained heads of houseflies (-1/usca domestica; approx. 2.5 heads/2 $\mathrm{ml}$ of saline in a Warburg vessel). In order to, study the inhibition by DINP, the desired amount of DDVP was added to the homogenate, a $\mathrm{CO}_{2}-N_{2}$ mixture was bubbled through for $5 \mathrm{~min}$, and the reaction mixture was then heated in a Warburg water-bath at 37 . Inzyme and inhibitor were then allowed to react for some time (incubation time), after which acctylcholine chloride solution was added from the side arm of the vessel (final concn. $0.015 . \mathrm{K}$ or $0.0075 . \mathrm{K}$ ). Readings were then taken at 10 min-intervals for at least one hour, though inhibition was nearly always calculated from the 30 -min readings. Incubation time was usually $1.5-2 \mathrm{~h}$, which in most cases proved to be sufficient for almost maximal inhibition.

References $p .6 I_{3}$. 


\section{RESULTS}

\section{The relation between inhibitor concentration and fractional inhibition}

In Fig. 2 the fractional inhibition $(\mathrm{I}-a)$ is plotted against the inhibitor concentration expressed in $I_{50}$ units $\left(I / I_{50}\right)$. It can easily be seen that the plots for the mouse-brain homogenate fit in quite well with the curve drawn for $\mathrm{I} \times a /(\mathrm{I}-a)=$ constant, whereas the plots for the fly-head suspension agree much better with the curve drawn for $I /(I-\alpha)=$ constant (i.e. inhibition is directly proportional to inhibitor concentration).

It seems justified to consider the inhibition of mouse-brain-ChE as slowly reversible and that of fly-head-ChE as irreversible. More direct evidence for this will be given below.

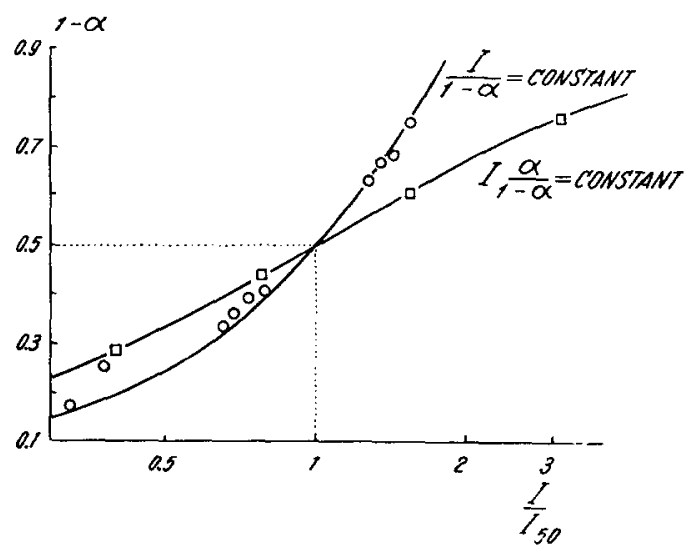

Fig. 2. Inhibition caused by different inhibitor concentrations. $O$ housefly-head homogenates; $\square$ mouse-brain homogenates.

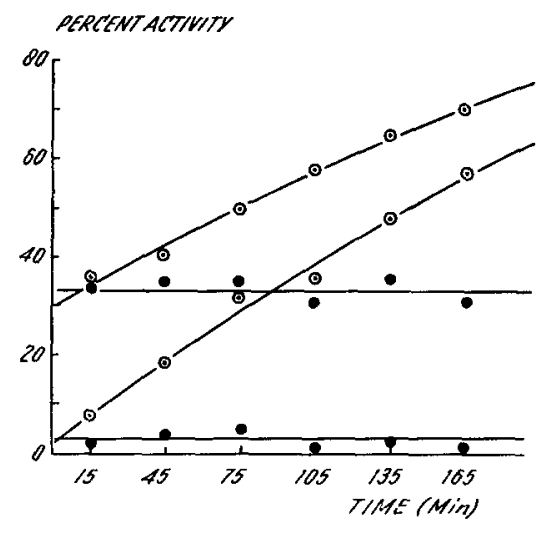

Fig. 3. Reactivution of ChE-activity. $\odot$ Mousebrain homogenates ( $10^{-7}$ and $5^{\cdot} 1^{-7} M$ DDVP). - Housefly-head homogenates $\left(7 \cdot 5 \cdot 10^{-10}\right.$ and $3 \cdot$ I $^{-9} M$ DDVP).

\section{Reversibility of inhibition}

Homogenates of mouse brain and fly heads were allowed to react with DDVP for $\mathrm{I} .5 \mathrm{~h}$. Then acetylcholine ( $\mathrm{ACh}$ ) was added from the side arm and manometer readings were taken at $30-\mathrm{min}$ intervals for as long as $3 \mathrm{~h}$. The velocity of AChhydrolysis during each 30 -min period could then be calculated and expressed as a percentage of the velocity in the corresponding control (non-inhibited) suspension. In Fig. 3 the percentage activity is plotted against time. It is evident that reactivation of the inhibited enzyme occurs only in the mouse-brain homogenates, which is in full agreement with the results described in the previous section of this paper.

According to the schematic picture, which has been outlined above (see Fig. I), the decrease of inhibition should proceed exponentially. In our view, the measurement and calculation of reaction velocity is not sufficiently accurate to allow of a conclusion as to whether our results satisfy this theoretical condition, though they are certainly not incompatible with it. The reactivation rate is rather low; about $2.5 \mathrm{~h}$ are required for $50 \%$ reactivation of the inhibited enzyme in the mouse-brain homogenates.

Similar experiments were performed with paraoxon (O,O-diethyl $O$ - $p$-nitrophenyl phosphate). No reactivation of inhibited enzyme could be observed, either in mousebrain homogenates or in fly-head homogenates. This is in full agreement with the 
findings of ALDRIDGE AND DAVISON ${ }^{2,5}$, who showed that diethylphosphorylated mammalian $\mathrm{ChE}$ is very stable, whereas the dimethylphosphorylated enzyme is hydrolysed and thus reactivated at a measurable though rather low rate (see also MYERS $^{6}$ ).

\section{Inhibition rate and affinity between enzyme and inhibitor}

The inhibition process could be followed by varying the incubation time, i.e. by exposing homogenates of mouse brain and fly heads to DDVP for different periods of time and measuring the resulting inhibition. This could be done by comparing the $\mathrm{ChE}$-activity in the first $30 \mathrm{~min}$ after the addition of the substrate with that of an uninhibited suspension. The reactivation of the mouse-brain-ChE during the $30 \mathrm{~min}$ required for the manometric determination is neglected and, in our view, is negligible. The results are shown in Figs. 4 and 5.

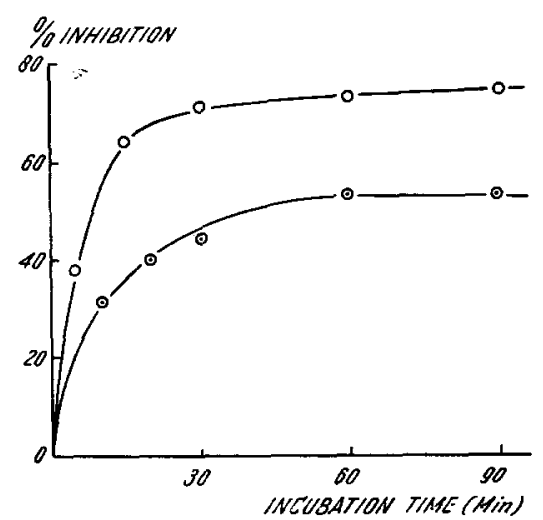

Fig. 4. Inhibition of mouse-brain-ChE. $\mathrm{O}-\mathrm{O} 6.8 \cdot 10^{-7} M$ DDVP; $\odot-\odot 1.7 \cdot 10^{-7} M$ DDVP.

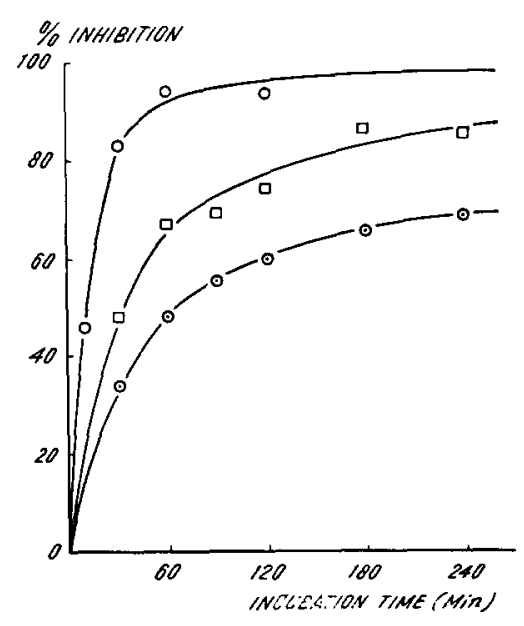

Fig. 5. Inhibition of housefly-head-ChE. $\mathrm{O}-\mathrm{O} 4 \cdot 10^{-8} M$ DDVP; $\square-\square 9 \cdot$ I $^{-10} M$ DDVP: $\odot-\odot 7 \cdot$ IO $^{-10} M$ DDVP.

It should be noted that the concentration of DDVP used in the experiments with fly-head homogenates are much lower than those used in the case of mouse-brain homogenates. At a rough estimate, equal initial rates are obtained with DDVPconcentrations that differ by a factor of about $5^{\circ}$ (for example: $1.7 \cdot$ IO $^{-7} M$ in the mouse-brain suspensions equals $4 \cdot$ IO $^{-9} M$ in the fly-head homogenates). Since, according to Fig. $\mathbf{I}$, the initial inhibition rate is quite independent of the stability of the phosphorylated enzyme, i.e. of the reversibility of the inhibition, it is clear that mouse-brain-ChE and fly-head-ChE are not only different with regard to this reversibility as described in section 2 , but must be different in other respects as well. This could be due to a difference in affinity ( $K_{i}$ mouse brain $>K_{i}$ fly heads) or to a difference in the velocity constant of reaction (2) $\left(k_{1}\right.$ mouse brain $<k_{1}$ fly heads), or, perhaps, to both.

So far we have assumed that the addition of $\mathrm{ACh}$ stops the inhibitory reaction. This is to be expected since $\mathrm{ACh}$ will readily react with the enzyme, thus considerably reducing the amount of free enzyme $(E)$ and consequently the concentration of the References p. 613 . 
enzyme-inhibitor complex (EI), which is determining the rate of reaction (2). That this assumption is quite justified, is brought out by the data included in Figs. 6 and 7 .

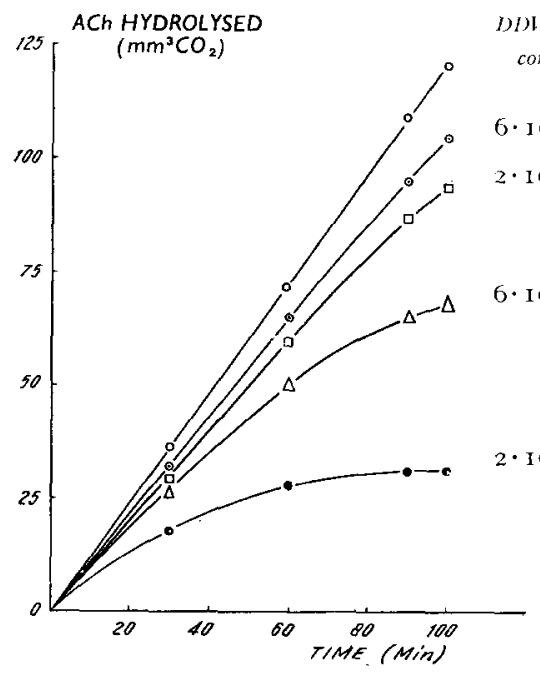

DII $P$-conc.

contro?

$\cdot 10^{-9} \mathrm{M} \quad 20 \% \quad 50 \%$

$10^{-8} M \quad 40 \% \quad 70 \%$

${ }_{10}^{-8} M \quad 75 \% \quad$ 100 $\%$

Fig. 6. Inhibition of fly-head-ChE by DDVP in the presence of substrate. DDVP added at zero time. Inhibition values in the table (right side) are calculated from the slopes of the curves at time go (see figure) and time 300 (not in the figure).

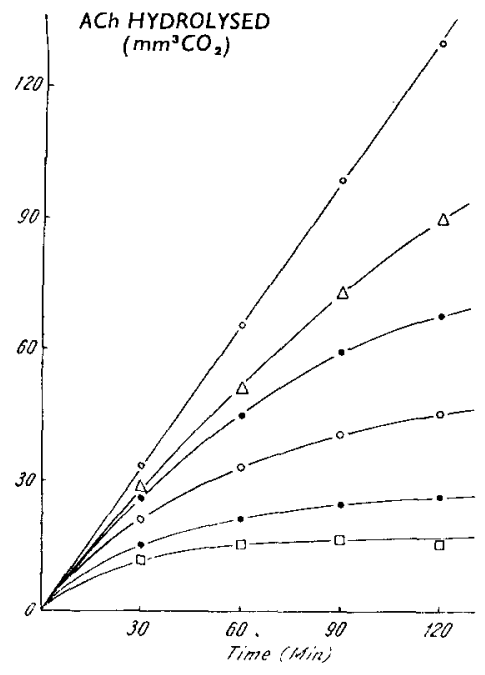

control

$2 \cdot 5 \cdot 10^{-6} M$

$5 \cdot 10^{-6} M$

$10^{-5} M$

$\cdot 10^{-5} M$

$4 \cdot 10^{-5} M$

Fig. 7. Inhibition of mouse-brain-ChE by DDVP in the presence of substrate. DDVP added at zero time. Inhibition values in the table (right side) are calculated from the slopes of the curves at time 90 (see figure) and time 300 (not in the figure).

These data were obtained by experiments in which DDVP was added about 35 min after the addition of $\mathrm{ACh}$. In this way the enzyme is protected by the substrate against attack by DDVP and the concentrations of DDVP required for inhibition are much ligher than they are in the absence of $\mathrm{ACh}$. When the dissociation constant $\left(K_{s}\right)$ of the enzyme-substrate complex is taken as $5 \cdot 10^{-4}$ (value given by AUGustinsSoN ${ }^{7}$ for mammalian true $\mathrm{ChE}$ ), whereas the substrate concentration in our experiments is approximately $\mathrm{I} .5 \cdot \mathrm{IO}^{-2} M$, it is clear that only a small fraction of the total enzyme will be available for reaction with DDVP. The inhibition rate, therefore, will Rejerences p. 6r3. 
be very low, unless the inhibitor concentrations are increased Io-roo-fold, as compared with those used in the absence of $\mathrm{ACh}$.

The results given in the Figs. 6 and 7 confirm our conclusion, that fly-head-ChE has much greater affinity to DDVP than mouse-brain-ChE. It can, moreover, be concluded that $1.5 \cdot 10^{-2} M A C h$ yields nearly full protection of the enzyme against DIVP-concentrations up to about $6 \cdot \mathrm{IO}^{-9} \mathrm{M}$ in the case of the fly-head homogenates and up to about $10^{-6} M$ in the case of the mouse-brain suspensions, at least during the first $30 \mathrm{~min}$, which is of great practical importance for the interpretation of the results of many of our experiments.

Remarkable differences between housefly-head and mouse-brain homogenates can be observed, when the inhibition values found after $\mathrm{I} .5 \mathrm{~h}$ and those found after $5 \mathrm{~h}$ are compared (see Figs. 6 and 7 ). In fly-head suspensions there is a considerable increase of the percentage inhibition between these two points of time, whereas in mouse-brain homogenates the inhibition value is nearly constant. This finding, in our view, agrees quite well with the idea that fly-head-ChE is irreversibly and mousebrain-ChE reversibly inhibited. As has been pointed out before, the addition of substrate will appreciably lower the concentration of free enzyme. In the case of an irreversible inhibition this will bring about a considerable decrease of the inhibition rate without, however, affecting the inhibition value finally reached. Any inhibitor concentration exceeding the enzyme concentration will, even if usually after a long time, result in $100 \%$ inhibition. It is reasonable, therefore, to expect a steady increase of the inhibition value, as is indeed found in the experiment with fly-head homogenates (Fig. 6). When, however, the phosphorylated enzyme is unstable, constant inhibition will occur as soon as the velocity of reaction (2) equals that of reaction (3), i.e. when $k_{1} \cdot[\mathrm{EI}]=k_{2} \cdot[\mathrm{EP}]$. The degree of inhibition (represented by [EP]) will depend on $[\mathrm{EI}]$. It will be clear from equation $(\mathrm{I})$, that at low concentrations of free enzyme as caused by the addition of substrate, high concentrations of the inhibitor will be required to obtain a certain concentration of EI and consequently a certain degree of inhibition. The results of the experiment with mouse-brain homogenates (Fig. 7) show, that constancy of the inhibition value is reached after about $\mathrm{I} .5 \mathrm{~h}$ and that actually much higher concentrations of DDVP are needed to obtain a certain degree of inhibition than in the absence of acetylcholine.

\section{Ratio of inhlibition rate and reversion rate}

Equation (6), which has been derived in the introduction to this paper, referred to the slopes of the curves 2 and 3 at times $t_{0}$ and $t_{1}$ in Fig. I. It should be noted, however, that these slopes represent respectively the initial inhibition rate $\left(\gamma_{i}\right)$ and the initial reversion rate $\left(r_{r}\right)$. So, equation (0) can be replaced by

$$
v_{r}=\alpha \cdot v_{i}
$$

This equation, of course, only applies to reversible inhibitions and only on condition that the concentration of the inhibitor undergoes no appreciable change.

It will be worth while investigating whether equation (7) holds for the inhibition of mouse-brain-ChE by DIDVP. The values for ${ }^{\prime} i$ and $i^{\prime} r$ can be derived from the Figs. 3 and 4 . When this is done for a DINYP-concentration that gives approximately $50 \%$ final inhibition and the velocities are expressed as percentages of the total amount of enzyme inhibited or reactivated per $30 \mathrm{~min}$, then $i^{\prime} i=90$ and $i^{\prime} r=\mathrm{s}$, while 
$\alpha=0.5(50 \%$ inhibition). It is evident, that equation ( 7$)$ in this case does not hold. This inevitably leads to the conclusion that the concentration of free inhibitor is considerably decreased during the experiments as may be clear from the following considerations.

Suppose the inhibition reaction to start at time o and a constant level of $50 \%$ inhibition to be reached at time $t$. Then, labeling the concentrations of the reacting compounds at times $o$ and $t$ by the adequate index, the following may be derived.

$$
\begin{aligned}
& v_{r}=k_{2} \cdot[\mathrm{EP}]_{t}=k_{1} \cdot[\mathrm{EI}]_{t} \\
& v_{i}=k_{1} \cdot[\mathrm{EI}]_{0}
\end{aligned}
$$

According to equation (I): $\quad \frac{v_{r}}{v_{i}}=\frac{[\mathrm{EI}]_{t}}{[\mathrm{EI}]_{0}}=\frac{[\mathrm{E}]_{t} \cdot[\mathrm{I}]_{t}}{[\mathrm{E}]_{0} \cdot[\mathrm{I}]_{0}}$

When the final inhibition is $50 \%$, then $\frac{v_{r}}{v_{i}}=\frac{8}{96}$ and $\frac{[\mathrm{E}]_{t}}{[\mathrm{E}]_{0}}=0.5$ and $\frac{[\mathrm{I}]_{t}}{[\mathrm{I}]_{0}}=\frac{\mathrm{I}}{6}$.

So, at time $t$ the inhibitor concentration in only $\mathrm{I} / 6$ of the initial inhibitor concentration. This value, however, should be considered as a very rough estimate only.

In equation (5) $[I]$ represents the inhibitor concentration actually present at the time when reactions (2) and (3) are proceeding at equal rates, i.e. $[\mathbf{I}]$ t. The theoretical considerations given in the first part of this paper thus led to the conclusion that, when certain conditions are fulfilled, $[\mathrm{I}]_{t} \cdot \alpha_{/}^{\prime}(\mathrm{I}-\alpha)$ is constant.

In section I (Fig. 2), however, I represents the total concentration of inhibitor initially present, i.e. $[\mathrm{I}]_{0}$, and our experimental results showed, therefore, that $[\mathrm{I}]_{\mathbf{0}} \cdot a /(\mathbf{I}-\boldsymbol{a})$ is constant.

In order to meet both the theoretical and the experimental requirements the amount of inhibitor which remains free and available for reaction with the enzyme $\left([\mathrm{I}]_{t}\right)$ must be directly proportional to the total amount of inhibitor added in the experiment $\left([\mathrm{I}]_{\mathbf{0}}\right)$.

This condition can be satisfied by assuming the formation of a reversible complex of DDVP and some compound, probably a protein; which is present in fairly great excess of the inhibitor. Up to now it has not been possible to furnish definite proof for this view, though the results of many experiments agree quite well with it. In a number of experiments mouse-brain homogenates were incubated for $\mathrm{I} .5 \mathrm{~h}$ with various concentrations of DDVP. Then fresh homogenates of fly heads were added and the inhibition of the fly-head-ChE resulting in each case was used for the calculation of the amount of free DDVP still present. The incubation of DDVP with mouse-brain suspensions actually caused a decrease of the concentration of free inhibitor. Seemingly this decrease did not exceed about $50 \%$ of the original inhibitor concentration, which is much less than the amount calculated above. This, however, is exactly what may be expected when the binding of DDVP is rever sible, since in this case the free inhibitor which is withdrawn from the solution by reaction with the $\mathrm{ChE}$ will be replaced by dissociation of the complex. The amount of DDVP available for reaction with the fly-head-ChE will thus be much larger than the amount of free DDVP present at any moment. Theoretically the formation of the reversible complex will only lower the inhibition rate without affecting the degree of inhibition finally reached. The decrease of fly-head-ChE inhibition values caused by previous incubation of the DDVP with mouse-brain homogenates will, therefore, certainly reflect the decrease of free inhibitor concentration without, however, furnishing an exact measure for it. 


\section{DISCUSSION}

In a previous paper ${ }^{1}$ the question was raised whether the difference in susceptibility to DDVP-inhibition between fly-head and mouse-brain homogenates must be ascribed to differences between the cholinesterases themselves or to differences with regard to other factors, e.g. binding of DDVP to other proteins. Experiments in which mixtures of mouse-brain and fly-head suspensions were exposed to DDVP led us to believe that it is very probable that considerable differences exist between the enzymes themselves. The analysis of the inhibition reactions given in the present paper enables us to deal with this problem in more detail. Several possibilities must be considered.

(I) Irreversible binding of DDVP to some compound in the mouse-brain homogenates could possibly eliminate a large part of the inhibitor, thus necessitating the use of much higher concentrations to obtain inhibition. This possibility can be ruled out on two considerations. In the first place it is very unprobable that, this being an important factor, the relation between the amount of inhibitor added and the inhibition value finally reached would accurately agree with that for a reversible inhibition as it actually does according to Fig. 2, in the case of mouse-brain homogenates. Secondly, such an irreversible binding by the mouse-brain homogenates is likely to exert a great influence on the inhibition of the fly-head-ChE in the combination experiments described in the last part of the previous section. As pointed out, the inhibition of the fly-head-ChE in these experiments was only slightly less than that found in the absence of mouse-brain.

(2) Reversible binding of DDVP to some compound in either homogenate is unable to explain a difference in susceptibility to DDVP, when the inhibition in both homogenates is irreversible. In that case the final inhibition value will theoretically be dependent only on the ratio of the amounts of enzyme and inhibitor. Reversible binding can, however, be an important factor in the case of a reversible inhibition since it considerably reduces the rate of the inhibitory reaction and thereby lowers the inhibition value at which equilibrium is reached between inhibition rate and reversion rate.

(3) Reversibility of the inhibition in the mouse-brain homogenates is likely to increase the inhibitor concentration required in order to obtain a certain degree of inhibition. However, in view of the rather low reversion rate as compared with the initial rate of the inhibitory reaction, the reversibility will certainly be insufficient for explaining the large difference in susceptibility found in our experiments.

(4) A difference in affinity between enzyme and inhibitor will, of course, give rise to very different rates of inhibition. If the inhibition process were irreversible in both cases this would not, however, affect the inhibition values finally reached. It will be clear from what has been said before that such a difference in affinity might be very important when one or both of the inhibition processes are reversible.

It has been the purpose of this paper to demonstrate that most probably, neither of the factors mentioned above will by itself give a satisfactory explanation of the large differences in susceptibility to DDVP. The analysis given in the preceding sections of this paper, however, has shown that three of the factors in co-operation are likely to give an appropriate explanation of the observed differences.

Summarizing, we may state that in our opinion the following three factors are important:

References $p .6{ }_{13}$. 
(a) Inhibition by DDVP is reversible in the case of mouse-brain-ChE and irreversible in the case of housefly-head-ChE.

(b) The affinity between fly-head-ChE and DDVP is much greater than that between mouse-brain-ChE and DDVP.

(c) In mouse-brain homogenates DDVP is reversibly bound to some compound, most probably a protein. Such binding may possibly occur also in fly-head homogenates, but if it does this will not affect the inhibition value finally reached.

The first two factors refer to differences between the enzymes themselves, whereas the third one refers to a possible difference in the chemical composition of the medium in which the reaction takes place.

In this discussion we have paid no attention to the possibility that the reversible inhibition of mouse-brain-ChE might be converted to an irreversible inactivation by a process more or less comparable to that described for DFP and sarin by J.INDORI: et al. ${ }^{8}$. So far, however, no indications were obtained that such a process could have affected our experimental results to an appreciable extent.

In addition to the investigations described in this paper, several experiments have been performed on the inhibition of horse-serum-ChE by DIDVP. The overall picture obtained with this preparation appeared to be remarkably similar to that observed in the case of mouse-brain homogenates. The inhibition proved to be slowly reversible. When the $\mathrm{ChE}$ of horse serum, mouse brain, and housefly heads are compared it appears that with respect to substrate specificity mouse-brain-ChE and fly-head-ChE are about similar' and distinctly different from the horse-serum-ChE, whereas with respect to reversibility of the inhibition by I)DVP, fly-head-ChE bohares quite differently from both types of mammalian- $\mathrm{Ch} \mathrm{E}$, tested.

\section{MRNOWJEDGEMENTS}

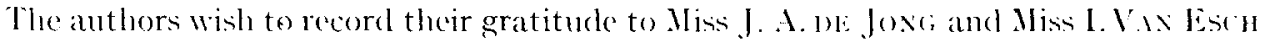
for their valuable assistance in the experimental work. They further take this opportunity of thanking Dr. B. L. Rothsters of Montrose Chemical Company (C.S.A.) for two gifts of 1$) 131$.

\section{STMMARY}

The paper deals with incestigations on the inhibition of the cholinesterases in mouse-brain and housefly-hed homogenates by (), ()-dimethyl 0-2, -dichlorovinyl phosphate (10121'). Its purpose is a explain the remarkable difference in susceptibility to 1 ) Sereral aspects of the inhilition process and the participating compounds were studied, such as (a) the relation beween the anount of inhibitor used and the percentage inhibition finally obtained, (li) 1 he reversibitity of the inhibition process and the rate of reversion, (c) the rate of the inhibitory

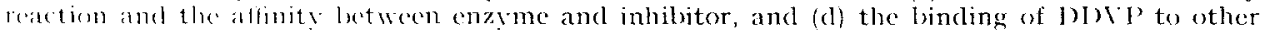
componmels. e.p. proteins, present in the homogenates. From the results it is coneluched that the

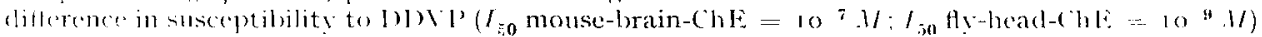
is mainly due lo thece co-c)erating factors: (I) Inhibition of mouse-brain-(

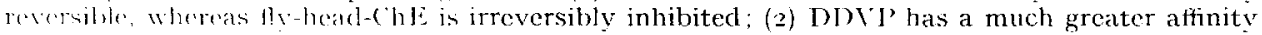

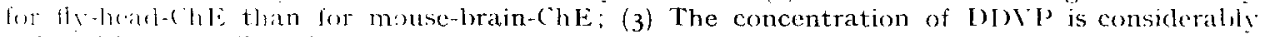
redeced le revesible binding to some compound present in the monse-brain bomogenates.

llorse-serum-(blialso prosed to be reversibly inhibited. The paper does not, howerer. contain quatititive datal on this preparation.

lieferences p. 613. 


\section{REFERENCES}

1 K. van Asperen, Mededel. Landbouwhogeschool en Opzoekingsstat. Staat Gent, 22 (1957) 477.

2 W. N. Aldridge, Biochen. J., 54 (I953) 442.

3 J. E. Casida, J. Agr. Food Chem., 4 (I956) 772.

4 I. Massart, in J. B. Sumner and K. Myrbäck, The Enzynes, Vol. I, Part I, Academic Press, New York, I950, p. 307.

5 W. N. Aldridge AND A. N. Davison, Biochem. J., 55 (r953) 763 .

6 D. K. MYers, Thesis, Iniversity of Amsterdam, I9.54.

7 K. B. Augustinsson, in J. B. Sumner and K. Myrb.̈.6, The Enzymes, Vol. I, Part I, Acalemic Press, New York, 1950, p. 443.

8 B. J. Jandorf, H. O. Michel, N. K. Schaffer, R. Egan and W. H. Summerson, Discussions Faraday Soc., 20 (195.5) I 34.

Received October 25th, I957

\section{PTERIDINES AS SUBSTRATES OF \\ MAMMALIAN XANTHINE OXIDASE}

\section{THE END-PRODUCT OF THE ENZYMIC OXIDATION \\ OF PTERIDINES*}

FEIIX BBERGMINN AND HANNA KWIETNY

Departnent of Pharmacology, The Hebrew University, Hadassah Medical School, Jerusalem (Israel)

The chemical and biochemical interrelationship of purines (I) and pteridines (II) has been recognised in the past.
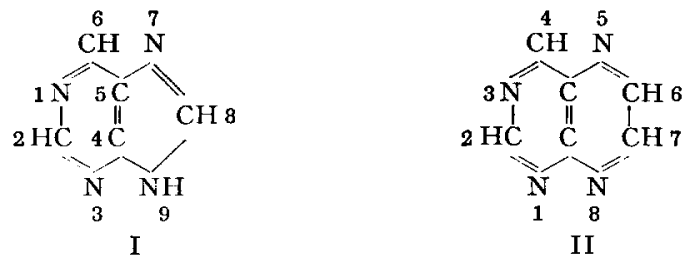

For cxample, Alsert ${ }^{1}$ has shown that on incubation of 2-hydroxypurine with glyoxal in apueous solution, 2-hydroxypteridine is formed, and that similar transformations can be carried out with other purines. ZIEGilir-GïNIER, SLuon .Nil)

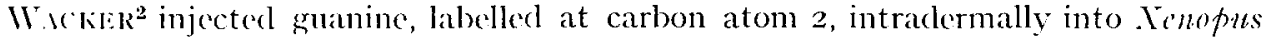
larvae and isolated afterwards labelled pteridines from their skin. These observations make it probable that in the cell certain metabolites are formed, such as $e^{2} \cdot 5^{2} \cdot 4,5$-diaminopyrimidines, which may undergo cyclisation to a variety of heterocyclic systems (pteridines, alloxazine $\left.\mathrm{s}^{3}\right)$.

All naturally occurring pterilines carry substituents in the 2- and +position of

* This investigation was supported ly a research grant of the Hadassah Mledical Organisation. The results reported form part of a l'l. i). thesis of 11 . Kwists, to be submitted to the laculty of Science, The Helnew lniversity, Jerusalem.

References P. 6 IS 